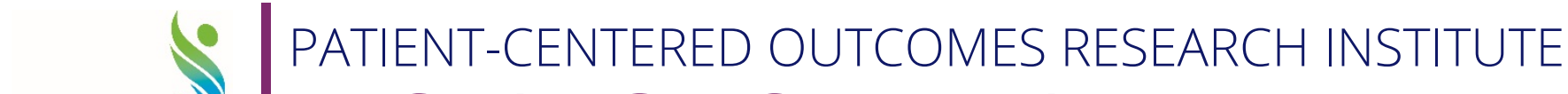 pcori ?. RESEARCH SUMMARY
}

\section{Comparing Two Diabetes Prevention Programs for American Indian and Alaska Native Adults in an Urban Community}

Principal investigator

Randall Scott Stafford, MD, PhD
Organization

Stanford University School of Medicine

\section{What was the research about?}

Type 2 diabetes is a long-term illness that causes blood sugar levels to rise. Keeping blood sugar levels normal can help prevent damage to the heart, brain, eyes, limbs, and kidneys. To reduce the risk for type 2 diabetes, people can eat healthy foods and reduce body fat by being active. American Indian and Alaska Native, or AIAN, adults are at high risk for type 2 diabetes. AIAN adults also are more likely than adults of other racial or ethnic groups to have mental health problems, such as depression and anxiety, which can make it hard to prevent diabetes.

In this study, the research team developed and tested a diabetes prevention program for AIAN adults that also addressed mental health. The team compared the new program with a standard program. Both were tailored to address the health needs of AIAN adults. Both involved 16 60- to 90-minute weekly group education sessions led by a trained health coach on diet, physical activity, and weight loss. The new program also included three ways to help people feel connected to others and improve mental health:

- Group discussions called talking circles, which promote social support

- Photography and photo review sessions to find the causes of health problems

- Sessions to share life stories that the team could post on social media
The research team looked at whether the new program reduced body mass index, or BMI, which measures body fat based on height and weight.

\section{What were the results?}

After one year, the new program wasn't better than the standard program at improving

- $\mathrm{BMI}$

- Quality of life

- Healthy eating

- How active people were

- Depression

- Confidence

In both programs, however, people who had more education sessions had larger reductions in BMI than those who had fewer sessions.

\section{Who was in the study?}

The study included 205 AIAN adults living in an urban area in California. Of these, 54 percent were also Hispanic. The average age was 52, and 79 percent were women. All were at high risk for getting type 2 diabetes based on their BMI, cholesterol, blood pressure, or blood sugar. 


\section{What did the research team do?}

The research team assigned people by chance to either the new or the standard program. The team checked BMI before and after the study. People also took a survey before the study started and again 6 and 12 months later. The survey asked about quality of life, healthy eating and exercise, depression, and confidence.

AIAN adults, caregivers, and health professionals helped the research team design the study and recruit people to take part in it.

\section{What were the limits of the study?}

This study took place in one city. Results may differ in other places or for AIAN adults living on reservations.
Of those in the new program, 18 percent went to all talking circles, 36 percent went to all photo review sessions, and only one person went to both sessions for sharing life stories. Results may differ if more people went to all parts of the new program.

Future studies could explore other ways to prevent type 2 diabetes for AIAN adults.

\section{How can people use the results?}

Doctors, nurses, and community partners can use the results of this study when considering ways to prevent type 2 diabetes among AIAN adults.

To learn more about this project, visit www.pcori.org/Stafford262. 